PROCEEDINGS OF THE

AMERICAN MATHEMATICAL SOCIETY

Volume 48, Number 2, April 1975

\title{
WEAK CHAINABILITY OF PSEUDOCONES
}

\author{
DAVID P. BELLAMY
}

\begin{abstract}
A pseudocone over $X$ is a compactification of $(0,1]$ with remainder $X . S$ is a circle. A characterization of those pseudocones over $S$ which are weakly chainable is given. (A continuum is weakly chainable if and only if it is a continuous image of the pseudoarc.) Covering projections and liftings are used, and a simple geometric interpretation of the result is that a pseudocone over $S$ is weakly chainable if and only if the absolute value of the winding number of any subarc of $(0,1]$ around $S$ is bounded by some $m>0$.
\end{abstract}

The following terminology will be used here. A continuum is a compact connected metric space. $I=[0,1] ; A=(0,1] ; S$ is the unit circle in the complex numbers. If $X$ is a continuum, a pseudocone over $X$ is a compactification of $A$ with remainder $X$ [1], [2], $R$ is the set of real numbers and $Z$ is the set of integers. A continuum $X$ is acyclic if every continuous map $f$ : $X \rightarrow S$ is nullhomotopic.

A. Lelek has introduced the notion of a weakly chainable continuum in [4]. The importance of these continua is that they are precisely the continuous images of the pseudoarc [4], [5]. In conversation, Sam B. Nadler, Jr. and J. Quinn have raised the question of which pseudocones over $S$ are weakly chainable. This is related to a problem posed by them in [6, Remark 6.2, p. 67]. It is the purpose of this note to provide an answer to this question. This is also of interest since Lelek's examples in [4, p. 28] are both pseudocones over circles. See also [3].

The definition of weak chainability is rather technical and is available in [4]. It is really unrelated to the present work, so it will not be given here. Instead, we use the following

Lemma. A continuum is weakly chainable if and only if it is a contin. uous image of a chainable continuum.

Received by the editors October 1, 1973 and, in revised form, February 27, 1974.

AMS (MOS) subject classifications (1970). Primary 54F20, 54F 15; Secondary 54C05, 54D35.

Key words and phrases. Compactification, covering projection, chainable continuum.

Copyright $\odot$ 1975. American Mathematical Society 
Proof. This is immediate from the Theorem in [4, p. 274] and Corollaries 1 and 3 of [4, p. 276].

Now, let $p: \mathbf{R} \rightarrow S$ be the usual covering projection; $p(t)=e^{i t}$. Let $P$ be a pseudocone over $S$, where $S \subseteq P$ and $j: A \rightarrow P$ is the embedding of $A$. Let $r: P \rightarrow S$ be a retraction. (One always exists by Proposition 1 of $[1$, p. 7] or Theorem 3 of $[1, \mathrm{p} .19]$.) Let $h: P \rightarrow S \times I$ be the embedding given by: $h(s)=(s, 0), s \in S ; h(j(s))=(r \circ j(s), s), s \in A$. Let $p \times 1: \mathbf{R} \times I \rightarrow S \times I$ be defined in the usual way. Then $p \times 1$ is a covering projection also. Let $Q=(p \times 1)^{-1}(h(p))$. Let $\left\{J_{k}\right\}_{k \in Z}$ be the set of all liftings of $r \circ j$ through $p$, where $0 \leq J_{0}(1)<2 \pi$, and where $J_{k+1}(t)=J_{k}(t)+2 \pi$ for each $t, k$.

Let $K_{k}: A \rightarrow R \times I$ be given by $K_{k}(t)=\left(J_{k}(t), t\right)$. Then $Q=R \times\{0\} \cup$ $\left[\bigcup_{k \in Z} K_{k}(A)\right]$. Now observe that the image $J_{k}(A)$ is a bounded subset of $R$ if and only if $K_{k}(A)$ is a bounded subset of $R \times I$ in the Euclidean metric. Also, if $J_{k}(A)$ is bounded for any $k$, it is for every $k$.

Theorem. Let $P$ be a pseudocone over $S$. Using the notation in the preceding discussion, $P$ is weakly chainable if and only if $J_{0}(A)$ is a bounded subset of $R$.

Proof. Suppose $J_{0}(A)$ is bounded. Let $X=\mathrm{Cl}\left(K_{0}(A)\right)$. Then $X$ is a pseudocone over a closed interval, and so is chainable by Lemma 2.7 of [2, p. 298]. Further, $(p \times 1)\left(\mathrm{Cl} K_{0}(A)\right)=h(P)$, so that, by the Lemma, $h(P)$ is weakly chainable. Thus $P$ is also weakly chainable.

Conversely, suppose $P$ is weakly chainable, let $X$ be a chainable continuum and let $f: X \rightarrow P$ be a continuous surjection. By the proof of Lemma 1 of $[7, \mathrm{pp} .74,75]$, there is a lifting $F: X \rightarrow \mathbf{R} \times I$ of $h \circ f$ through $p \times 1$. Then $F(X) \subseteq Q$, since $(p \times 1)(F(X))=h(P)$.

Since $K_{k}(1) \in F(X)$ for some $k$, we may choose $F$ so that $K_{0}(1) \in F(X)$. Then $K_{0}(t) \in F(X)$ for all $t \in A$; for if $K_{0}(t) \notin F(X)$ for some $t<1$, then $M=\left\{K_{0}(t): t>x\right\}$ is a nonempty proper closed and open subset of $F(X)$, contradicting the connectedness of $X . \quad\left(M \neq \varnothing\right.$, since $K_{0}(1) \in M ; M \neq F(X)$, since $F(X) \cap(R \times\{0\}) \neq \varnothing$, while $M \cap(R \times\{0\})=\varnothing . M$ is open and closed in $F(X)$ since

$$
\begin{aligned}
M & =\left\{\left(J_{0}(t)+s, t\right):-\epsilon<s<\epsilon ; 1 \geq t>x\right\} \cap F(X) \\
& =\left\{\left(J_{0}(t)+s, t\right):-\epsilon \leq s \leq \epsilon ; 1 \geq t \geq x\right\} \cap F(X)
\end{aligned}
$$

where $0<\epsilon<2 \pi$.)

Thus, we must have $K_{0}(A) \subseteq F(X)$. Since $F(X)$ is compact, it is bounded in $R \times I$. Thus $K_{0}(A)$ is bounded and, hence, so is $J_{0}(A)$. 
Corollary. A pseudocone $P$ over $S$ is weakly chainable if and only if it is a continuous image of some acyclic continuum.

Proof. If $P$ is weakly chainable it is a continuous image of a chainable continuum which is acyclic. If $F: X \rightarrow P$ is a continuous surjection, where $X$ is acyclic, then, again, by the proof of Lemma 1 of $[7, \mathrm{pp} .74,75]$, there is a lifting of $F$ of $h \circ f$ through $p \times 1$ (using the above notation). Again, without loss of generality, $K_{0}(A) \subset F(X)$ and, hence, $K_{0}(A)$ is bounded. Thus, $J_{0}(A)$ is bounded and $P$ is weakly chainable.

There is a simple geometric interpretation of the above Theorem. $P$ is weakly chainable if and only if there exists an $m>0$ such that for any $s, t$ $\epsilon A$, where $s<t$, the absolute value of the "winding number" of $h \circ j \mid[s, t]$ is strictly less than $m$. (Intuitively, it is clear what "winding number" means here-the net algebraic number of times which $h \circ j$ wraps $[s, t]$ around $S$ in $S \times I$. To define "winding number" precisely here, we shall have to return to the lifting $J_{0}$ and define the winding number to be, say, $\left(J_{0}(s)-J_{0}(t)\right) / 2 \pi$. It would suffice to consider only those $s, t$ for which this is an integer.)

\section{REFERENCES}

1. D. P. Bellamy, Topological properties of compactifications of a half open interval, Ph.D. Thesis, Michigan State University, East Lansing, Mich., 1968.

2. An uncountable collection of chainable continua, Trans. Amer. Math. Soc. 160 (1971), 297-304. MR 44 \#3292.

3. M. K. Fort, Jr., Images of plane continua, Amer. J. Math. 81 (1959), 541546. MR 21 \#5173.

4. A. Lelek, On weakly chainable continua, Fund. Math. 51 (1962/63), 271282. MR $26 \# 742$.

5. J. Mioduszewski, A functional conception of snake-like continua, Fund. Math. 51 (1962/63), 179-189. MR 26 \#1859.

6. Sam B. Nadler, Jr. and J. Quinn, Embeddability and structure properties of real curves, Mem. Amer. Math. Soc. No. 125 (1972).

7. Edwin H. Spanier, Algebraic topology, McGraw-Hill, New York, 1966. MR $35 \# 1007$.

DEPARTMENT OF MATHEMATICS, UNIVERSITY OF DELAWARE, NEWARK, DELAWARE 19711 